\title{
(Un)doing masculinity in der Kita
}

\author{
Tobias Bockstaller
}

\subsection{Erwartungen an Männlichkeiten in der Kita}

Männliche Fachkräfte in Kindertagesstätten sind bezogen auf die Herstellung männlicher Geschlechtsidentität - doing gender - in ihrem Arbeitsalltag mit besonderen Herausforderungen konfrontiert. Der Beruf der Fachperson Betreuung wird in der Schweiz einerseits aufgrund der geringen Anzahl männlicher Fachkräfte und andererseits aufgrund der weiblichen Konnotation der Arbeitsinhalte sowie der Geschichte des Berufs als typischer Frauenberuf betrachtet. Vor diesem Hintergrund werden an Männer im Beruf verschiedene Erwartungen gerichtet (Buschmeyer 2012). Erstens sollen sie ein männliches Element in einen weiblich konnotierten Arbeitsbereich einbringen. Es wird von ihnen erwartet, dass sie sich stereotyp männlich verhalten und sich zum Beispiel für das Auswechseln der Glühbirnen zuständig erklären. Zweitens sollen sie sich als Identifikationsfigur insbesondere für die Jungen anbieten. Es wird angenommen, dass männliche Identifikationsfiguren den Jungen im weiblichen Berufsfeld fehlen. Drittens wird erwartet, dass männliche Fachkräfte eine väterliche Rolle einnehmen (Buschmeyer 2012, S. 43), wodurch ein traditionelles Familienbild in die Kita gebracht wird. Diese Erwartungen können große Herausforderungen für männlichen Fachkräfte darstellen. Sie vermitteln Annahmen über eine geschlechterdifferenzierende Arbeitsteilung und können somit als wichtiger Aspekt des doing gender interpretiert werden (Wilz 2002).

Doing gender findet zum Beispiel statt, wenn ausschließlich die männliche Fachperson in einem mehrheitlich weiblichen Team vor allem männlich konnotierte Aufgaben übernehmen muss (Rohrmann et al. 2010). Während diese

T. Bockstaller $(\bowtie)$

Bern, Schweiz 
Tätigkeiten in Einrichtungen, in denen keine Männer arbeiten, selbstverständlich von Frauen ausgeführt werden, verändert sich dies häufig, sobald ein Mann in die Einrichtung kommt (Rohrmann 2006). Dabei wird eine bestimmte Tätigkeit - wie das oben genannte Beispiel des Wechselns einer Glühbirne - erst dann zu einer männlich konnotierten Tätigkeit, wenn eine männliche Fachperson in die Einrichtung kommt. Doing masculinity heißt in diesem Fall das Ausführen von Tätigkeiten, die dem männlichen Stereotyp entsprechen.

Nach Connell (2015) findet hier doing masculinity durch zwei Aspekte statt: Einerseits wird Männlichkeit in einen Gegensatz zu Weiblichkeit gebracht. Der männliche Betreuer muss etwas grundlegend anderes tun, als die Mehrheit der weiblichen Mitarbeiterinnen, um männlich zu bleiben. Andererseits sind es explizit stereotyp männliche Tätigkeiten und Aspekte, die hier betont werden. Es sind hegemoniale Männlichkeitskonstruktionen, die in den Handlungen referenziert werden, während alternative Männlichkeiten mehrheitlich unthematisiert bleiben.

Doing gender und doing masculinity werden als etwas beschrieben, das in der Interaktion zwischen Menschen hergestellt wird. Diese Herstellung findet kaum bewusst statt und wird häufig auch nicht als solche wahrgenommen (Gildemeister 2017, S. 2f.). In diesem Kapitel wird doing masculinity in körperlichen Praktiken untersucht. Auch Männlichkeiten werden nur dann reproduziert, wenn Menschen interagieren. Dabei ist eine Interaktion nicht nur als mündlicher Austausch zwischen zwei anwesenden Menschen zu verstehen, sondern kann auch die Interaktion eines Menschen mit einer Gruppe, einer fiktiven Person (zum Beispiel auf einem Werbeplakat), mit einem Raum, mit Objekten oder mit einer beobachtenden, forschenden Person sein. Gildemeister (2017, S. 5) definiert doing gender als ,die intersubjektive Validierung in Interaktionsprozessen durch ein situationsadäquates Verhalten und Handeln im Lichte normativer Vorgaben und unter Berücksichtigung der Tätigkeiten, welche der in Anspruch genommenen Geschlechtskategorie angemessen sind". Entscheidend für die Herstellung von Männlichkeiten ist also das, was die Person macht. Aufgrund dessen, wurden in der in diesem Kapitel beschriebenen Studie die körperlichen Praktiken männlicher Fachpersonen in den Blick genommen.

\subsection{Hegemoniale, alternative und komplizenhafte Männlichkeiten}

Connell (2015) geht in ihrem 1995 erstmals erschienenen Buch Masculinities (deutsch Der gemachte Mann) davon aus, dass es nicht eine, sondern verschiedene Formen von Männlichkeit gibt. Sie definiert Männlichkeit als ,[...] eine 
Position im Geschlechterverhältnis; die Praktiken, durch die Männer und Frauen diese Position einnehmen, und die Auswirkungen dieser Praktiken auf die körperliche Erfahrung, auf Persönlichkeit und Kultur" (Connell 2015, S. 124). In ihrer Theorie der hegemonialen Männlichkeit zeigt Connell (2015, S. 124), dass es nicht nur ein Machtungleichgewicht zwischen Mann und Frau gibt, sondern auch zwischen verschiedenen Männlichkeiten. Die hegemoniale Männlichkeit, die sich an traditionellen Rollenbildern - weiß, heterosexuell, gesund, vollzeitbeschäftigt, Fokus auf Macht und Karriere - orientiert, steht dabei in der Hierarchie zuoberst. Diese hegemoniale Männlichkeit ist aber veränderbar und unterscheidet sich auch lokal und global. Sie zeichnet sich dadurch aus, dass sie in der Struktur der Geschlechterverhältnisse die bestimmende Position einnimmt.

Connell (2015) ordnet der hegemonialen Männlichkeit verschiedene andere Männlichkeiten unter, wie die homosexuelle, die komplizenhafte und die marginalisierte. Die homosexuelle Männlichkeit wird häufig mit Weiblichkeit gleichgesetzt und findet sich am unteren Ende der Hierarchie der Männlichkeiten. Komplizenhafte Männlichkeit beschreibt eine Form von Männlichkeit, die zwar äußerlich hegemoniale Männlichkeit zeigt, sich aber nach innen nicht so verhält. Diese Form von Männlichkeit kommt sehr häufig vor, da sich nur wenige männliche Personen vollständig der hegemonialen Männlichkeit zuordnen lassen, aber viele diesem Ideal nacheifern. Die marginalisierte Männlichkeit entsteht dort, wo Männlichkeit mit anderen diskriminierenden Faktoren wie Race oder Class in Beziehung tritt und dadurch für die hierarchische Position weniger wichtig wird. Meuser (2006) und Buschmeyer (2012) führen beide aus, dass Connells Konzept hegemonialer Männlichkeit eine gewisse Unschärfe aufweist. Auch für diese Untersuchung ist es wichtig, hegemoniale Männlichkeit nicht nur als Ausdruck einer bestimmten Körperlichkeit zu verstehen, sondern als generatives Prinzip. Dies bedeutet, dass man hegemoniale Männlichkeit zu nicht-hegemonialen Männlichkeiten in Relation stellen muss (Meuser 2006, S. 164). Es geht also nicht darum, hegemoniale Männlichkeit als Folge gewisser körperlicher Ausdrücke zu verstehen (zum Beispiel breitbeiniges Sitzen als Darstellung hegemonialer Männlichkeit), sondern sie als soziale Praxis zu beschreiben, der eine Palette an verschiedenen möglichen Ausdrucksweisen zur Verfügung steht (hegemoniale Männlichkeit herzustellen, erfolgt unter anderem durch breitbeiniges Sitzen, es ist aber nur eine von verschiedenen Möglichkeiten).

Eine Forschungsarbeit, die einer vergleichbaren Fragestellung nachgeht, ist die bereits zitierte Arbeit von Buschmeyer (2012). Die Autorin zeigt in ihrer Untersuchung in deutschen Kindergärten zwei unterschiedliche Männlichkeitstypen auf. Einerseits einen komplizenhaften und andererseits einen alternativen Typus. Der 
komplizenhafte Typus übernimmt wo nötig, die ihm zugeschriebenen stereotypen Tätigkeiten und wird dadurch als weniger konflikthaft erlebt. Der alternative Typus hingegen muss sich in seinem Alltag stärker mit seiner eigenen Männlichkeit auseinandersetzen und setzt sich gegen stereotype Zuschreibungen zur Wehr. Buschmeyer (2012, S. 274) stellt jedoch auch fest, dass die Zuordnung zu einem der zwei Typen sich durchaus im Verlauf einer Berufskarriere verändern kann. Auch steht der Männlichkeitstypus mit der Art der Institutionen im Zusammenhang: Während in staatlichen Institutionen eher männliche Fachpersonen des komplizenhaften Typus arbeiten, können alternative Männlichkeitsformen eher in Institutionen gefunden werden, die aus Bürgerinitiativen entstanden sind. Ausgehend davon, dass hegemoniale Männlichkeit Berührungen zwischen Kindern und Männern generell problematisiert und als potenziell gefährlich darstellt, können sich bezogen zum Beispiel auf die körperliche Nähe zu den Kindern die beiden Typen darin unterscheiden, dass der Komplizenhafte zwar körperliche Nähe zulässt, dabei aber ganz klare Grenzen vorgibt und wenn immer möglich versucht, diese Nähe zu verhindern. Der alternative Typus hingegen setzt die körperliche Nähe situativ bewusst ein (Buschmeyer 2012, S. 271; Tab. 7.1).

In ihrer Dissertation ,Symbolische Väter, junge Wilde und professionelle Pädagogen" hat Tennhoff (2018) auf Grundlage des auch hier verwendeten Materials und in Ergänzung der in Kap. 6 vorliegenden Analyse untersucht, welche Subjektivierungsweisen männliche Fachpersonen in Schweizer Kindertagesstätten zur Herstellung von Männlichkeiten zeigen. Sie wertete Interviews mit den männlichen Fachkräften und den Leitungspersonen der Kindertagesstätten aus. Tennhoff (2018, S. 248) zeigt auf, dass es im Diskurs über männliche Fachkräfte bezüglich der Argumentation, weshalb mehr männliche Personen als Fachpersonen Betreuung arbeiten sollten, zwei Hauptpositionen gibt. Die erste Position sieht männliche Fachkräfte, ausgehend von einem traditionellen Familienbild, als Familienersatz im Sinn der Komplementarität zu weiblichen Fachkräften. Die zweite Position definiert männliche Fachkräfte als Professionelle, als Erneuerer und Verbesserer der Arbeit in den Kitas. Bei den Subjektivierungspraktiken der männlichen

Tab. 7.1 Beispiel für Typen von Männlichkeiten in Handlungen nach Buschmeyer (2012)

\begin{tabular}{l|l|l|l}
\hline & Hegemonial & Komplizenhaft & Alternativ \\
\hline Körperkontakt & - Arbeitet nicht in & - Lässt Nähe zu & - Setzt Körperkontakt \\
& einem Beruf mit & - Setzt klare Grenzen \\
& Körperkontakt & $-\begin{array}{l}\text { Verhindert Kontakt, } \\
\text { wenn immer möglich }\end{array}$ & Situation ein \\
& & & \\
\hline
\end{tabular}


Fachkräfte stellt Tennhoff (2018) fest, dass diese weitaus vielfältiger und wechselhafter sind als angenommen. So können männliche Kinderbetreuende gleichzeitig verschiedenen Positionen einnehmen. Auf der von ihr untersuchten diskursiven Ebene lässt sich keine alternative Männlichkeit im Sinn von Buschmeyer (2012) finden (Tennhoff 2018, S. 250). Bei männlichen Fachpersonen finden sich aber immer Momente, in denen Elemente hegemonialer Männlichkeit sichtbar werden. Professionalisierung hat für männliche und weibliche Fachkräfte in Kindertagesstätten nicht die gleiche Bedeutung. Aufgrund der weiblichen Konnotation des Berufs müssen weibliche Fachpersonen Professionalität nicht inszenieren, da sie diese qua Geschlecht bereits besitzen. Männliche Fachpersonen hingegen inszenieren sich über Professionalität und grenzen sich dadurch von anderen, weniger professionellen Betreuungspersonen ab (Tennhoff 2018, S. 251f.).

Buschmeyer (2012, S. 264) geht davon aus, dass hegemoniale Männlichkeit in einem weiblich konnotierten Beruf gar nicht existiert, sondern ihr einzig nachgeeifert werden kann, da hegemoniale Männlichkeit sich wie beschrieben von Weiblichkeit abzugrenzen hat. So lange Betreuungsarbeit gesellschaftlich als weiblich konnotiert wird, ist es entsprechend nicht möglich, bei der Ausübung dieser Tätigkeit hegemoniale Männlichkeit herzustellen. Dies bedeutet jedoch nicht, dass nicht hegemonialer Männlichkeit nachgeeifert werden kann. Wie ihre Untersuchung aufzeigt, gibt es unterschiedliche Möglichkeiten, wie und wo sich dieses Nacheifern ausdrücken kann.

Aufgrund der Untersuchungen von Buschmeyer (2012) und Tennhoff (2018) ist davon auszugehen, dass es eine intersektionale und konfigurative Betrachtungsweise der Herstellung von Männlichkeiten braucht (Meuser 2006, S. 170). Anstatt der Zuteilung der Fachpersonen zu fixen Männlichkeitstypen ist es angebrachter, die Typen fluid unterschiedlichen Handlungsmustern zuzuordnen. Entsprechend rückt der Kontext, der die Möglichkeiten der körperlichen Praktiken beeinflusst, in den Fokus.

\subsection{Bedeutung körperlicher Praktiken in der Herstellung sozialer Praxis}

Um die Konstruktion von Männlichkeit theoretisch fundiert untersuchen zu können, muss neben Connells (2015) Konzept der hegemonialen Männlichkeit auf weitere Konzepte zurückgegriffen werden. Hinsichtlich des hier gewählten Fokus auf körperliche Praktiken bietet sich das Habituskonzept Bourdieus an. Beide Konzepte gehen davon aus, dass Geschlecht sozial konstruiert wird und somit im sozialen Alltag verankert ist. Diese sozialen Praktiken drücken sich unter anderem 
in körperlichen Tätigkeiten aus. Weiter gehen beide davon aus, dass Geschlecht kultur- und milieubedingte Ausprägungen aufweist (Brandes 2004).

Unter Zuhilfenahme von Bourdieu lassen sich auch Institutionen und Strukturen als Hintergrund und Ursache von Interaktionsprozessen verstehen (Gildemeister 2017). Mit dem Habituskonzept wird der Fokus stärker auf die Stabilität und Beharrlichkeit von bestimmten Konstruktionsweisen oder Männlichkeitsvorstellungen gelegt. Das Habituskonzept geht davon aus, dass die Dualismen in der Geschlechterordnung tief in Dingen und Körpern verankert sind und nicht auf ihrer rein sprachlichen Thematisierung beruhen. Entsprechend schwer sind sie zu dekonstruieren und aufzuheben (Bourdieu 2012, S. 178). Sie führen zu RegelmäBigkeiten der physischen und sozialen Ordnung (Bourdieu 2012, S. 46). Bourdieu (2012, S. 63) nennt diese Strukturen ,symbolische Ordnung“. Es sind die Wahrnehmungs-, Denk- und Handlungsmatrizen aller Mitglieder der Gesellschaft. Individuen handeln nicht nur rational. Sie haben gelernt, sich in inkorporierten Strukturen zu bewegen, die sie als natürlich empfinden (Buschmeyer 2012, S. 59). Dies bedeutet, dass das Individuum emotional nicht unterscheiden kann, ob es sich bei einer Eigenschaft um eine strukturell erlernte oder um eine genetisch bedingte handelt. Es kann also davon ausgegangen werden, dass diese inkorporierten Strukturen in jeder Interaktion mit anderen Menschen zum Ausdruck kommen - unabhängig davon, ob sich das Individuum dessen bewusst ist oder nicht.

Körperliche Praktiken können vor diesem Hintergrund als „verleiblichte“ soziale Praxis verstanden werden (Brandes 2004). Im Habitus äußern sich gesellschaftliche Strukturen, die als körpereigen wahrgenommen werden (Buschmeyer 2012). Brandes (2004) führt dazu aus, dass die körpernahe Dimension des männlichen Habitus nur eine Dimension des Konstrukts Männlichkeit ist: „Diese Dimension ist den Subjekten nur begrenzt bewusst zugänglich und berührt das Gefühl zum eigenen Körper ebenso wie Geschmack, intellektuelle Vorlieben und praktische Neigungen“ (Brandes 2004, S. 5). Auch wenn der Habitus nicht determiniert, was das Individuum in einer Situation tut, definiert er die Grenzen des jeweiligen Handelns. Die körperlichen Praktiken, die einem bestimmten Habitus folgen, nennt Bourdieu „Hexeis“. Besonders wichtig ist es also, die beobachteten körperlichen Praktiken immer in Abhängigkeit zum Kontext zu verstehen. Dabei spielt sowohl das Setting, in dem sich eine Person momentan befindet, wie auch die Lebenswelt, die für den Habitus prägend ist, eine Rolle. Für die männliche Fachperson in der Kita bedeutet das, dass die Praktiken einerseits von den momentan vorhandenen Handlungsmöglichkeiten und andererseits von den erlernten Handlungsmustern aufgrund des Settings beeinflusst werden. Darauf aufbauend werden in dieser Untersuchung vor allem die Hexis untersucht, d. h. die 
körperlichen Praktiken. Rückschlüsse auf den dahinterstehenden Habitus lassen sich aufgrund der Handlungsergebnisse vermuten. Den Überlegungen von Brandes (2004) folgend, werden die Männlichkeitstypen von Connell (2015) in dieser Untersuchung als Varianten eines männlichen Habitus verstanden.

Körperliche Praktiken von Fachpersonen in der Interaktion mit Kindern haben bezüglich der Vermittlung von Geschlechterwissen einen besonderen Stellenwert. Die Fachpersonen erzeugen mit ihren Praktiken einen Habitus, der sie in ihrer täglichen Arbeit beeinflusst und sie geben ihn aufgrund ihrer Vorbildfunktion als Erziehende auch an die Kinder weiter. Buschmeyer (2012, S. 127) nennt dies einen ,[...] im doppelten Sinne [...] vergeschlechtlichten Habitus“. Der Einfluss der körperlichen Praktiken der Fachpersonen auf die Kinder ist somit ein wichtiger Faktor von doing und undoing gender im Sinn der Reproduktion bzw. der Veränderung von Geschlechtskonstruktionen. Dies gilt entsprechend auch für doing masculinity, also das Herstellen von Männlichkeiten - in diesem Fall durch männliche Fachpersonen im weiblich konnotierten Berufsalltag.

Aufgrund der beschriebenen Relevanz körperlicher Praktiken zur Herstellung von Geschlechtsidentität wurde die Forschungsfrage folgendermaßen konkretisiert: Wie und in welchen Kontexten manifestiert sich hegemoniale Männlichkeit in den körperlichen Praktiken männlicher Fachkräfte in Kindertagesstätten? Obwohl es unterschiedliche Praktiken der Herstellung hegemonialer Männlichkeit gibt, ist davon auszugehen, dass die Praktiken stabilen Voraussetzungen folgen (wie zum Beispiel der hierarchischen Position). Sie müssen fluide und situativ hergestellt werden und werden somit vom Kontext beeinflusst.

Im folgenden Abschnitt wird anhand des Forschungsdesigns konkret ausgeführt, wie die hegemoniale Männlichkeit und der Kontext, in der sie hergestellt wird, operationalisiert werden.

\subsection{Rekonstruktive videobasierte Beobachtung}

Visuelles Datenmaterial gewinnt in der ethnomethodologisch ausgerichteten Forschung immer mehr an Bedeutung, da es eine präzise Rekonstruktion sozialer Praxis ermöglicht (Blaschke 2012, S. 58). Am meisten zu hinterfragen ist dabei nach Blaschke (2012) die Bedeutung der Anwesenheit der Kamera. Welchen Einfluss hat sie auf die Konstruktion sozialer Praktiken? Auch Wagner-Willi (2013, S. 133) stellt fest, dass der videogestützten Beobachtung, die mit der teilnehmenden Beobachtung verwandt ist, eine immer größere Bedeutung zukommt. Sie sieht die Vorteile der videobasierten Beobachtung auch in ihrer Reproduzierbarkeit und dem Detaillierungsgrad der mikroskopischen Perspektive auf soziale Wirklichkeit, 
die hierdurch ermöglicht wird. Auch Wagner-Willi stellt fest, dass Videomaterial kein genaues Abbild der Realität darstellt. So findet ein Wechsel statt von einem drei- auf ein zweidimensionales Bild und Aspekte wie Gerüche gehen verloren. Die Bilder werden von den Forschenden mitkonstruiert, sie bestimmen, welcher Teil der Realität auf welche Art und Weise gezeigt wird (Wagner-Willi 2013, S. 149). Wie auch andere Methoden der qualitativen Sozialforschung sind Analysen von videobasierten Beobachtungen also keinem Objektivitätsstreben im klassischen Sinn zuzuschreiben. Aufgrund dieses Mangels an Objektivität und der guten Eignung des Materials, die Performativität der sozialen Alltagswirklichkeit aufzuzeigen, eignet sich die videobasierte Beobachtung besonders gut, um ethnomethodologische Untersuchungen durchzuführen (Wagner-Willi 2013, S. 150).

Unter körperlichen Praktiken werden in der Untersuchung alle Interaktionen verstanden, die eine körperliche Aktivität einschließen. Sprachliche Interaktionen (Gespräche) werden somit nicht analysiert. Sprachliche Interaktion, die gleichzeitig mit den körperlichen Praktiken stattfinden, werden als zu den körperlichen Tätigkeiten dazugehörend betrachtet.

Um die Herstellung von Männlichkeiten in den körperlichen Praktiken männlicher Fachkräfte zu untersuchen, wurden 50 Stunden Videodaten zu den insgesamt sieben männlichen Fachpersonen in den vier untersuchten Kitas codiert und analysiert. Es handelt sich bei den beobachteten Personen um ausgebildete Fachpersonen Betreuung. Zwar wurde das Material vom Autor nicht selbst vor Ort erhoben, dank der mehrdimensionalen Daten der Videos (bewegtes Bild und Ton) konnte dennoch im Vergleich zum Beispiel mit Tonaufnahmen näher am Alltag der Fachpersonen ausgewertet werden. Das Wegfallen des persönlichen Bezugs zum Forschungsfeld erwies sich für die Analyse des Habitus als Vorteil, da das Material ohne Erfahrungen aus der Erhebung und entsprechend ohne Einfluss der Interaktionen mit den untersuchten Personen im Feld betrachtet werden konnte. Es ist davon auszugehen, dass dank des Wegfallens des persönlichen Bezugs zu den Forschungsobjekten objektiver am Material gearbeitet werden konnte. Ein weiterer Vorteil des Videomaterials für die Untersuchung von körperlichen Praktiken im Vergleich zum Beispiel zu einer teilnehmenden Beobachtung ist, dass das Material mehrmals betrachtet werden konnte und somit Notizen mehrfach reflektiert und überprüft werden konnten.

Um die Videobeobachtungen auszuwerten, wurden sie zuerst mit dem Programm Atlas.ti aufbereitet und analysiert. In einem ersten Schritt wurde das gesamte Videomaterial anhand der bereits vorgenommenen Codierung nach Geschlecht der Betreuungspersonen sortiert. In diesem ersten Schritt konnten alle 
Tab. 7.2 Exemplarische Codegruppe

\begin{tabular}{l|l}
\hline Codegruppe & Dazugehörende Codes \\
\hline An-/Zurechtweisen & Anweisen \\
& Beruhigen \\
& Festhalten \\
& Schimpfen \\
& Streit schlichten \\
& Stuhl wegnehmen \\
& Wegnehmen \\
& Zurechtweisen \\
\hline
\end{tabular}

600 Sequenzen, in denen eine männliche Fachperson zu sehen ist, ausgewählt werden. Allen ausgewählten Personen wurden anonymisierte Namen zugewiesen. Im nächsten Schritt wurden die Sequenzen nach Personen codiert, um festzulegen, welche Person in der jeweiligen Sequenz zu sehen ist. In einer ersten Codierphase wurden alle Sequenzen nach den darin ausgeführten körperlichen Praktiken codiert, dabei ergaben sich Codes wie zum Beispiel „Holz hacken“ oder „Am Arm halten“. Wie man am Beispiel der Tab. 7.2 sieht, wurden dann aus allen gefundenen Codes aufgrund ihrer Ähnlichkeit Codegruppen erstellt, die im darauffolgenden Schritt auch als Kategorien, um die Handlungen der untersuchten Personen innerhalb der Gruppen zu vergleichen, verwendet werden konnten.

In einem letzten Schritt wurden zu allen codierten Sequenzen Memos mit den beobachteten körperlichen Praktiken erstellt, die einerseits die Ausführung der Handlungen beschreiben und anderseits zum Teil bereits theoretische Überlegungen enthalten.

Exemplarisches Memo: Reto kniet sich zu dem Mädchen, das Holzhacken will, hin. Er schaut ihr zu, um zu sehen, ob es geht. Dabei hält er selbst das Stück Holz - ohne Angst davor, dass sie ihn hackt. Er lobt sie als es gut klappt. Er schaut, dass das Setting immer stimmt, nimmt etwas Holz vor ihren Füßen weg, damit sie nicht stolpert. Er rückt das Beil zurecht, als sie es falsch hält. Das Kind hackt drauf los und trifft fast den Finger von Reto, er bleibt ruhig und sagt nur, da habe er Schwein gehabt. Er bezieht die Schuld auf sich selbst und sagt, er habe nicht aufgepasst. Dies lässt darauf schließen, dass Reto genau weiß, was er tut. Durch das genaue Einhalten seiner Vorgaben kann das Kind eine gefährliche Tätigkeit ausführen. Doing masculinity? Er lässt das Kind kontrolliert etwas Risikoreiches tun und bringt damit auch sich selbst in Gefahr.

Jede der untersuchten männlichen Fachpersonen wurde auf diese Weise zunächst hinsichtlich der identifizierten körperlichen Praktiken beschrieben und in einem zweiten Schritt auf die dabei gezeigten Elemente hegemonialer Männlichkeit hin analysiert. Die dabei als Elemente hegemonialer Männlichkeit betrachteten 
Praktiken (welche Praktiken sind Ausdruck eines traditionellen Männlichkeitsverständnisses?) sind in Tab. 7.3 nach Codegruppen geordnet dargestellt. Welche Praktiken als hegemonial männlich definiert wurden, ist unter anderem abhängig vom gesellschaftlich geformten Habitus des Forschenden, von biografischen Faktoren und auch von situativen Erlebnissen, wie das über den gesamten Analyseprozess geführte Forschungstagebuch zeigt. Im Forschungstagebuch wurden regelmäßig sowohl das Forschungsdesign als auch die Rolle des Forschers reflektiert. Wiederholt wurde dabei festgestellt, dass das subjektive Erleben hegemonialer Männlichkeit im Alltag die Wahrnehmung in unterschiedlichen Situationen beeinflusst: So zum Beispiel, dass die Begegnung mit männlichen Fachpersonen, die einen ausgeprägt hegemonialen Ausdruck zeigen, die Wahrnehmung der Handlungen aller Fachpersonen in der Analyse beeinflussen kann. Oder dass das Erleben von als stark hegemonial empfundenen Personen im Alltag die Auslegung davon, welche körperlichen Praktiken als hegemonial männlich eingestuft werden, beeinflussen. Diese Dynamik muss bei Darstellung und Interpretation der Ergebnisse stets mitbedacht werden. Die Vorstellungen davon, welche Praktiken als hegemonial eingestuft werden, sind eng verbunden mit der Sozialisation des Forschers in einer von bestimmten hegemonialen Männlichkeitsbildern geprägten Gesellschaft. Die Tab. 7.3 soll darum möglichst genau aufzeigen, welche Elemente in dieser Untersuchung als hegemonial männlich definiert und codiert wurden.

Anhand der definierten Handlungsdimensionen (Codegruppen) wie zum Beispiel dem „Trösten“ oder dem „Zurechtweisen“ wurden sieben Fachpersonen verglichen, um Rückschlüsse auf inhaltliche Sinnzusammenhänge machen zu können (Kelle und Kluge 2010, S. 91f.). Zusätzlich zu den Fachpersonen untereinander wurden die körperlichen Praktiken der Fachpersonen auch im Kontext ihrer jeweiligen Kita betrachtet.

Der Vergleich der Fälle fand über den gesamten Analyseprozess hinweg statt. Verschiedene Faktoren - wie das pädagogische Konzept (wie ermöglichen die Fachpersonen Lernsituationen, welche Lernmomente für die Kinder lässt das Setting zu), die Fehlerkultur (wie wird reagiert, wenn sich Kinder nicht wie gewünscht verhalten), Körperberührungen (wo und wann finden Berührungen statt) und der Körperausdruck (wie bewegen sich die Fachpersonen, welche Gestik benutzen sie etc.) der männlichen Fachpersonen, die die Konstruktion von Männlichkeiten beeinflussen, wurden fallübergreifend verglichen. 


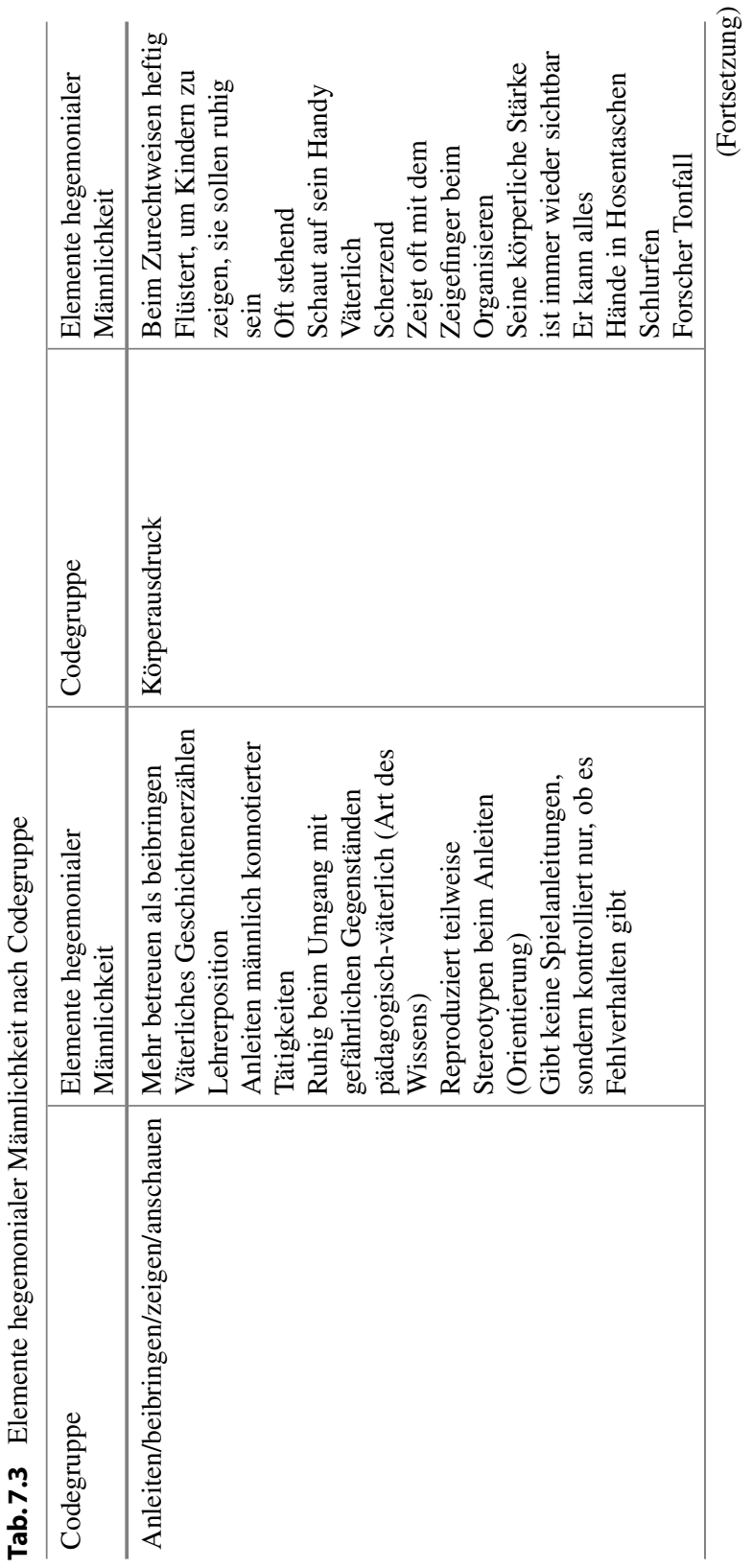




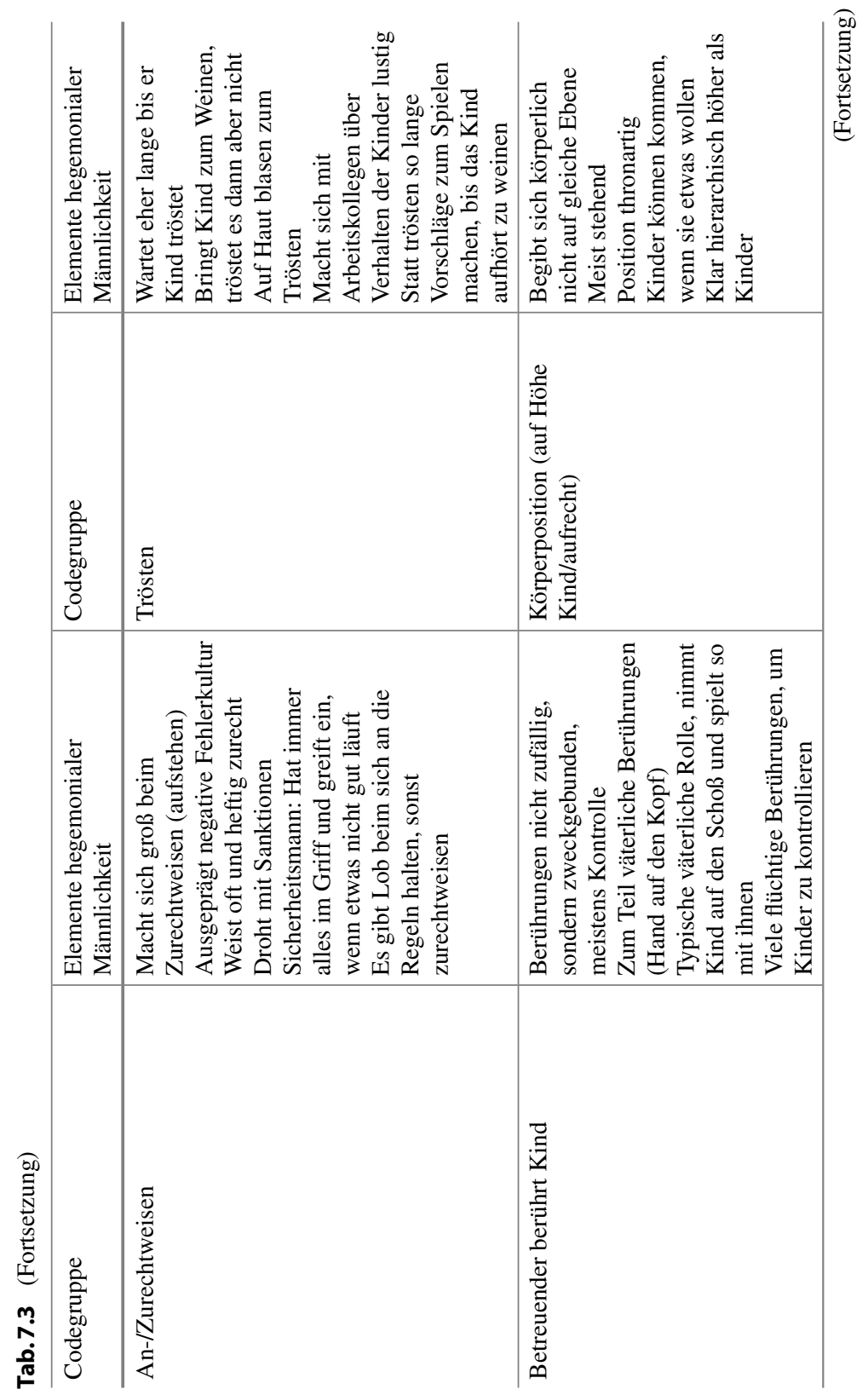




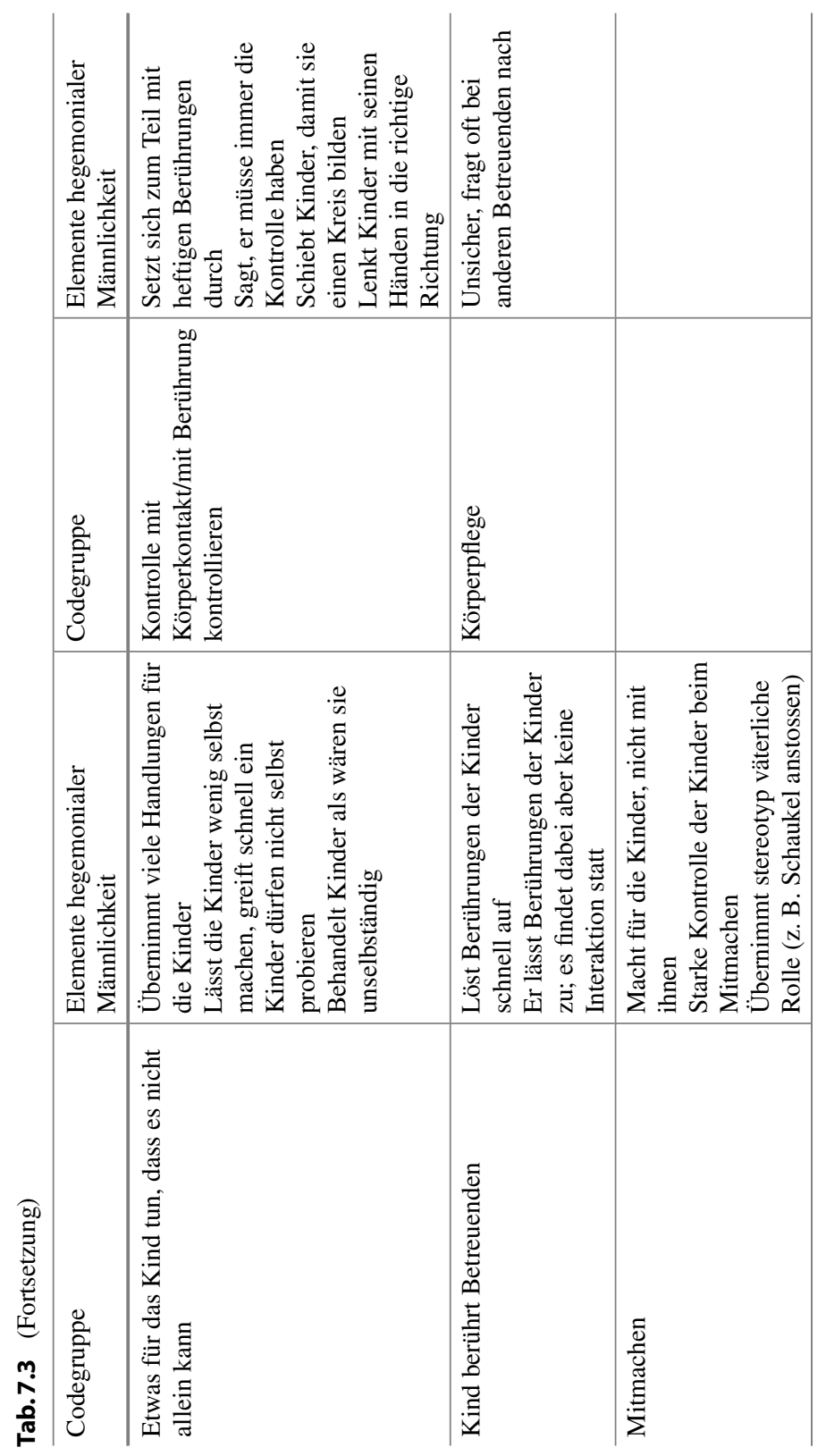




\subsection{Die Konstruktion hegemonialer Männlichkeit in körperlichen Praktiken}

Im folgenden Abschnitt werden die körperlichen Praktiken von vier der insgesamt sieben analysierten männlichen Fachpersonen genauer beschrieben und in einem zweiten Schritt miteinander verglichen. Wie werden verschiedene Männlichkeiten konstruiert und welchen Männlichkeitstypen lassen sich die einzelnen Beobachtungen bzw. Situationen zuordnen? Wie schon mehrfach angedeutet, kann keiner der vorliegenden Fachpersonen einem genauen Männlichkeitstypus zugeordnet werden. Vielmehr zeigen alle Männer in ihren körperlichen Praktiken unterschiedliche Männlichkeitskonstruktionen.

\subsubsection{Vier Kinderbetreuer und ihre körperlichen Praktiken}

\section{Michel}

Am meisten fällt bei Michel auf, dass er viel Körperkontakt mit den Kindern hat. Der Körperkontakt scheint bei ihm immer einen bestimmten Zweck, ein bestimmtes Ziel zu verfolgen. So nutzt Michel zum Beispiel Körperkontakt in angeleiteten Einheiten wie einem Kreis, in dem die Kinder mit den Betreuenden sitzen und gemeinsam singen. Dort nimmt er Kinder, die sich nicht an die Regeln des Kreises halten, die unruhig sind oder andere stören, zu sich. Dafür hebt er sie auf, hält sie bei sich auf dem Schoß oder setzt sie neben sich. Dies mit dem Ziel, die Kinder unter Kontrolle zu halten und sie dazu zu bringen, an der Einheit teilzunehmen oder diese zumindest nicht zu stören. Wenn die Einheit von einer anderen betreuenden Person angeleitet wird, übernimmt Michel spezifisch die Aufgabe, störende Kinder zu kontrollieren. Zufällige Berührungen finden bei Michel nicht statt. Meist geht es darum, ein Kind zu kontrollieren, indem er es zum Beispiel am Arm hält, um ihm zu zeigen, dass es nicht weglaufen soll. Folgender Memoausschnitt fasst dies zusammen:

Die Kinder rennen herum. Michel stoppt sie mit der Hand. Er fängt sie ein und zieht sie zum Teil grob am Arm. Er geht für die Zurechtweisung nicht herunter, sondern bleibt stehen. Er stellt die Ordnung her und weist die Kinder mit der Hand auf die Matratzen. Während er vorher so entspannt war, möchte er nun, dass die Kinder sich möglichst schnell auf die Siesta vorbereiten. Dafür legt er selbst Hand an, trägt Kinder herum und zieht sie um. Er hält ein Kind im Schoß fest, als es nicht folgen will, und trägt es dann auf einen Stuhl, um es von der Situation zu trennen (Memo vom 27.04.2018). 
Körperberührungen zwischen Michel und den Kindern gehen fast ausschließlich von Michel aus. Möchte ein Kind Michel von sich aus zum Beispiel umarmen, so löst er diese Berührung schnell auf. Das häufige Zurechtweisen der Kinder, bei dem Michel viel Körperkontakt einsetzt, scheint mit dem in den Situationen kaum sichtbaren pädagogischen Konzept seiner Kita zusammenzuhängen. So bekommt man den Eindruck, dass Michel sich beim Zurechtweisen der Kinder nicht wirklich wohl fühlt und dies auch nicht gern macht. Er scheint häufig dazu gezwungen, die Kinder zu kontrollieren, da sich diese oft nicht an die geforderten Ordnungen halten und diese immer wieder verlassen oder stören. Die geforderte Ordnung entspricht dabei oft nicht dem Bedürfnis der Kinder, sondern mehr einem Erwachsenenwunsch nach Kontrolle. Entsprechend ist es immer wieder, die Aktivitäten der Kinder innerhalb dieser Vorgaben zu halten. Ein weiteres Beispiel dafür ist das Memo einer Situation, in der die Betreuenden mit den Kindern im Kreis sitzen:

Kreissituation. Michel holt die Kinder mit der Hand zurück. Die Situation funktioniert nicht. Michel nimmt zwei auffällige Kinder auf den Schoß. Michel hat Mühe, den Kreis allein durchzuführen. Er leitet den Kreis so an und singt mit den Kindern. Er muss die Kinder sehr viel zurechtweisen, weil sie nicht mitmachen. In der Art und Weise aber nicht doing masculinity. Er setzt sich nicht durch. Er zieht sein Singen durch, auch wenn die Kinder nicht mitmachen (Memo vom 27.04.2018).

Die geforderte Ordnung muss von den Betreuenden immer wieder hergestellt werden und Michel ist sehr darum bemüht. Dies erfordert häufige Interventionen. Zumeist handelt es sich dabei nur um ein kurzes Hinweisen der Kinder auf eine gewünschte Verhaltensveränderung, ohne danach zu kontrollieren, ob die Kinder dies auch umsetzen. Oft muss sich Michel sogleich der nächsten Situation zuwenden. Trotz dieser hektisch wirkenden Situationen bleibt Michel meist ganz ruhig. Er kann sich in diesen unruhigen Situationen gut bewegen und kommt nur ganz selten aus dieser Ruhe aus. Tut er dies, so verändert sich sein Verhalten komplett und er wirkt dabei für einen kurzen Moment forsch.

Was bei Michel auffällt ist, dass er, sobald er mit den Kindern arbeitet, auf dem Boden sitzt. Dabei ist er zwar mit den Kindern auf Augenhöhe gleichzeitig aber weniger mobil, da die Kinder ja stehen. Muss er eine Situation klären, steht er meistens dafür auf. Das Handeln von Michel mit den Kindern ist geprägt von Betreuung. Situationen mit einem durch Beobachtung erkennbaren pädagogischen Gehalt kommen seltener vor. Die meisten Tätigkeiten werden von Michel zur Unterstützung der Kinder ausgeführt. Es ist auffällig, wie wenig selbstständig die Kinder um Michel herum sind. Sie dürfen nur selten versuchen, etwas selbst zu machen. So zieht Michel viele Kinder um, ohne sie dabei versuchen zu lassen, 
sich beispielsweise den Pullover selbst auszuziehen. Meistens legitimiert er diese Handlungen vor den Kindern, indem er zum Beispiel darauf hinweist, dass sie jetzt Zähne putzen oder Schlafen gehen und daher schnell umgezogen werden müssen. Bei allen pflegerischen Tätigkeiten zeigt Michel große Professionalität und Effizienz. Seine Handgriffe sitzen und werden rasch und mit größtmöglichem Komfort für die Kinder ausgeführt.

Muss ein Kind getröstet werden, zeigt Michel viel Fürsorglichkeit. Weinende Kinder werden von Michel auf den Schoß genommen und er gibt ihnen so lange Nähe, bis sie sich beruhigt haben. Zumeist wartet er ab, bis sich das Kind beruhigt hat und es findet kein erkennbares, aktives Bearbeiten der auslösenden Situation statt. Auffallend ist, dass sich Michel situativ sehr unterschiedlich verhält. Trägt er die Verantwortung für die Situation mit den Kindern, so lässt er sich weniger auf Spiele und Gespräche ein. Umgekehrt ist er hierfür sehr offen, sobald er die Situation nicht leiten muss.

\section{Sebastian}

Die körperlichen Handlungen von Sebastian folgen der Strukturiertheit der Kita, in der er arbeitet. Er bewegt sich innerhalb eines pädagogisch gestalteten Settings. Die Kinder der Kita sind verhältnismäßig selbstständig, entsprechend wenig müssen sie von Sebastian unterstützt werden. Er bietet seine Hilfe immer erst an, nachdem ein Kind erfolglos versucht hat, eine Tätigkeit selbst auszuführen. Er vermittelt aber nicht den Eindruck dies zu tun, weil er zum Beispiel unaufmerksam ist, sondern viel mehr, weil er den Kindern möglichst viele Situationen bieten möchte zu lernen möglichst selbständig zu sein. Sebastian ist bemüht, aus jeder Situation eine Lernsituation für das Kind zu schaffen. So müssen die Kinder zuerst selbst versuchen, sich anzuziehen, bevor Sebastian eingreift und ihnen hilft. Ein kleines Kind, dass noch nicht gut gehen kann, muss es immer zuerst selbst versuchen, bevor Sebastian ihm die Hand zur Hilfe anbietet. Es gibt wenig Konfliktsituationen in den untersuchten Sequenzen, entsprechend selten muss Sebastian zurechtweisend eingreifen. Muss er dies tun, so bietet er dem Kind auch alternative Handlungsmöglichkeiten an. Ein Kind stört ein älteres Kind beim Malen. Statt es wegzuschicken, gibt Sebastian ihm eine dem Alter entsprechende Möglichkeit, auch zu malen. Bei Sebastian wird in den Situationen sehr deutlich, dass er auf die Bedürfnisse der Kinder eingeht und auch bemüht ist, sie jeweils umzusetzen. Er erscheint empathisch, hört den Kindern zu und scheint sie gut lesen zu können. Muss er einem Kind etwas wegnehmen oder es zurechtweisen, so erklärt er immer genau, was er tut. So sagt er einem Kind zuerst: „Ich nehme dir jetzt den Pinsel weg“, bevor er es dann tatsächlich macht. 
Körperliche Berührungen kommen zwischen Sebastian und den Kindern selten vor. Wenn er ein Kind berührt, geschieht dies häufig, um seine Aussage zu verstärken. So sagt er einem Kind, man müsse nun mit dem Aufräumen beginnen und berührt es am Ende seiner Anweisung am Hinterkopf. Damit zeigt er auch körperlich, dass das Aufräumen nun beginnt. Je kleiner und unselbstständiger die Kinder sind, desto mehr Berührungen lässt Sebastian zu. Dies passt zum beobachteten pädagogischen Vorgehen: ein großes Spiel- und Lernangebot, in dem sich die Kinder möglichst selbstständig bewegen sollen. Sebastian erzählt den Kindern häufig Geschichten. Dabei nimmt er eine Rolle ein, die als typisch großväterlich bezeichnet werden kann: Er sitzt auf einem Stuhl vor den Kindern und erzählt gestenreich aus einem Buch. Dabei interagiert er immer wieder mit den Kindern und lässt auch Berührungen vonseiten der Kinder zu. In solchen Situationen muss er die Kinder häufiger zurechtweisen. Dabei scheint aber das Ziel im Vordergrund zu stehen, alle Kinder einzubeziehen. Zum Beispiel soll sich ein Kind wieder hinsetzen, damit alle anderen das Buch auch sehen können, aus dem er erzählt. Wie man im folgenden Memoausschnitt sieht, scheint er ein besonderes Interesse an der motorischen Entwicklung der Kinder zu haben und bietet ihnen immer wieder die Möglichkeit, zu üben:

\begin{abstract}
Sebastian hilft in dieser Sequenz einem Kind, das noch nicht gut laufen kann, aufzustehen und hält es dann an der Hand. Er hält es an beiden Händen, damit es gehen kann. Er scheint Freude an diesem Beibringen zu haben. Er versucht das Kind so gut wie möglich allein laufen zu lassen. Er kümmert sich nur noch um dieses Kind und dreht eine Runde mit ihm. Er lobt es immer wieder. Mit einem Praktikanten lassen sie das Kind vom einen, zum anderen gehen. Zwei Männer sitzen am Boden und das Kind läuft hin und her. Sie scheinen fasziniert vom Gehenlernen und lassen dem Kind viel Zeit. Sebastian versucht das Kind so wenig wie möglich zu halten (ein Finger), damit es selbst stehen lernt (Memo vom 27.04.2018).
\end{abstract}

Die Kinder dürfen in Sebastians Gegenwart potenziell gefährliche Gegenstände wie Messer oder Scheren benutzen. Dafür werden sie zuerst von Sebastian genau instruiert, wie sie diese benutzen sollen, dürfen sie dann aber auch ohne Aufsicht gebrauchen. Sebastian begibt sich nur selten auf die Höhe der Kinder, sondern steht meistens oder sitzt auf einem Stuhl. Auch wenn er so nicht auf Augenhöhe mit den Kindern ist, erweckt er doch allein durch seine Art, mit den Kindern zu sprechen, das Gefühl, dass er die Kinder gleichberechtigt behandelt. Dies zeigt sich auch in Situationen, in denen er Kinder tröstet. Er spendet dort nur so viel Zuneigung wie nötig und versucht, das Kind die Situation selbst meistern zu lassen. 


\section{Reto}

Alle beobachteten körperlichen Praktiken finden bei Reto im Freien statt. Reto zeichnet sich durch eine ausgeprägt positive Fehlerkultur aus. Aus einem Fehlverhalten oder Unvermögen eines Kindes stellt er immer eine positive Situation für das Kind her. Als zum Beispiel zwei Kinder mit langen Ästen andere Kinder stören, die in ein Spiel mit Wasser an einem Brunnen vertieft sind, interveniert Reto, indem er sie darauf hinweist, diese nicht zu stören. Auf diese Zurechtweisung folgt aber sofort die Empfehlung, mit den Ästen gemeinsam ein Fechtspiel zu machen. Im folgenden Memoausschnitt ist ein Junge beim Holzhacken aufgrund seiner Kraft nicht fähig, ein Holzstück zu spalten. Reto leitet ihn an und gibt ihm dabei deutlich zu verstehen, dass es kein Problem sei, dies nicht zu schaffen:

Reto bereitet weiter Holz vor, setzt sich hin und zeigt einem Jungen, wie er spalten muss. Er hilft ihm dabei, indem er das Holz für ihn hält. Er erklärt immer wieder, wie er es machen muss. Er nimmt es, wie bei anderen Sequenzen auch, sehr ernst, um in der Risikosituation möglichst viele Gefahren auszuschließen. Er sagt einem anderen Kind, es solle Abstand halten. Er motiviert den Jungen, fester zu hacken. Er geht auf positive Art und Weise mit dem Unvermögen des Kindes um. Das Kind hat nicht das Gefühl, dass es das nicht kann (Memo vom 27.04.2018).

Reto begründet und kommentiert seine Handlungen durchweg. Je nach Situation hat er hierfür jedoch weniger Zeit. Insbesondere in Übergangssituationen, zum Beispiel beim Aufbruch aus dem Wald zurück zur Kita, weist er die Kinder häufiger als sonst zurecht und reagiert weniger pädagogisch, als das sonst der Fall zu sein scheint. Schnell vorwärtszukommen ist dann das wichtigste Ziel, dem er anderes unterordnet. Fast jede andere Situation wird von Reto zu einer pädagogisch wertvollen gemacht, die Kinder können bei ihm immer etwas lernen. Er nimmt sich viel Zeit, auch einzelnen Kindern Aufmerksamkeit zu geben und ihnen etwas beizubringen. Dabei liegt sein Fokus auf der Förderung der Selbstständigkeit der Kinder. Die Kinder sollen bei ihm lernen, es selbst zu können. So insistiert er in einer Sequenz im Wald, dass die Kinder sich selbst orientieren sollen. Er geht lange mit ihnen durch den Wald und interveniert nicht. Schließlich findet die Gruppe andere Kinder, durch die sie Informationen zur Orientierung erhalten. Reto leitet im Wald viele als stereotyp männlich identifizierbare Aktivitäten wie Holz hacken, schnitzen oder sich orientieren an. Er hat keine Bedenken, die Kinder Messer oder Axt benutzen zu lassen und leitet sie dabei auf das Bedürfnis des jeweiligen Kindes achtend an. Berührungen kommen nur selten vor. Wenn Reto ein Kind berührt, dient es meist dazu, eine seiner 
Aussagen zu unterstreichen. Er berührt Kinder zum Beispiel, indem er ihnen die Hand auf den Kopf legt. Berührungen der Kinder lässt Reto vor allem in Lernsituationen zu. Zum Beispiel übermittelt er einigen Kindern am Boden sitzend Informationen zu Insekten. Die Kinder dürfen ihm dabei körperlich nahekommen und beim Zuhören auf seinen Beinen sitzen. Den Kindern wird zugleich nur geholfen, wenn sie etwas nicht selbst machen können. Ohne die Aufforderung eines Kindes hilft Reto nicht. Manchmal fragt er nach, ob ein Kind Hilfe möchte und hilft im Falle einer Zustimmung vonseiten des Kindes. Hinsichtlich des Körperausdrucks fällt bei Reto auf, dass er häufig mit dem Zeigefinger auf Menschen und Dinge zeigt. Er scherzt oft mit den Kindern und beginnt auch einmal spontan zu singen, als sie gemeinsam durch den Wald gehen. In pflegerischen Situationen zeigt Reto Sicherheit und Professionalität. Er hat die Kinder stets im Blick und kontrolliert immer wieder einmal die Temperatur oder die Windel eines Kindes. Er lässt sich ab und zu auf ein Spiel mit den Kindern ein, vor allem in Situationen, in denen Kinder besondere Aufmerksamkeit brauchen. Beim Trösten zeigt Reto die Haltung, dass Kinder möglichst viel eigenständig machen sollen und bringt einem Kind falls nötig viel Aufmerksamkeit und Fürsorglichkeit entgegen.

\section{Timo}

Timos Körperhaltung erscheint leger. Sein Gang kann als schlendernd beschrieben werden. Oft zeigt er sich in geschlossenen Positionen, zum Beispiel mit den Händen in den Hosentaschen oder mit verschränkten Armen. Er nutzt seinen Zeigefinger als Ordnungsinstrument und schließt seine Aussagen oft mit dem Wort „gut“ oder „so“ ab, vermutlich um zu zeigen, dass Situationen für ihn nun beendet sind und es nun mit etwas anderem weitergeht. Er passt seinen Tonfall der Situation an, flüstert zum Beispiel, wenn die Kinder ruhig sein sollen oder benutzt einen forschen Ton, wenn ihm etwas nicht gefällt.

Timo weist die Kinder zurecht, er spricht konkrete Sanktionen aus (siehe Memo) und setzt diese dann auch durch. Er handelt scheinbar ungeduldig und zeitweise grob. Lob erhalten die Kinder von Timo, wenn sie sich an die Regeln halten. Diese Art der Fehlerkultur funktioniert beim Lösen der beobachteten Konflikte jedoch meistens nicht, scheinbar gelöste Konflikte flammen schon nach kurzer Zeit zwischen den Kindern wieder auf. Dieser Ausschnitt aus einem Memo zeigt die Fehlerkultur gegenüber den Kindern auf, die Timo in allen beobachteten Momenten zeigt

Timo kniet zu einem Kind herunter. Er sagt, es dürfe keine Steine werfen und weil es dies getan habe, dürfe es heute nicht mehr auf die Schaukel. Eine Bestrafung. Er 
zeigt dem Kind auf, was es für andere Möglichkeiten habe. Das Kind schaut zu Boden. Timo sagt: „Da bin ich“, um die Aufmerksamkeit des Kindes zu haben. Er fragt was es tun möchte und zwingt es, sich zu entscheiden. Es soll sich zwischen zwei Optionen entscheiden. Es entscheidet sich und er schickt es, auf die Aktivität zeigend, weg (Memo vom 27.04.2018).

Timo funktioniert als Betreuer. Es konnten wenige Situationen beobachtet werden, in denen er den Kindern etwas beizubringen scheint. Meist sitzt er inmitten der spielenden Kinder und hilft, falls ein Kind danach verlangt, oder greift ein, wenn etwas nicht gut läuft. Die angeleiteten Spiele scheinen eng begleitet werden zu müssen, da es den Kindern oft schwerzufallen scheint, sich an die Regeln zu halten.

Pflegerische Situationen werden von Timo professionell und routiniert ausgeführt, ohne die Kinder dabei zur Selbstständigkeit zu ermutigen. Timo cremt die Kinder ein, auch wenn sie dies, so scheint es, selbst machen könnten. Auch das Naseputzen und das Händewaschen wird nicht von den Kindern selbstständig ausgeführt. Die Kinder müssen sich beim Händewaschen vor das Waschbecken stellen, Timo hebt sie dann hoch und hält ihre Hände unter das fließende Wasser. Danach stellt er sie wieder zurück auf den Boden. Timo berührt die Kinder häufig mit flüchtigen Berührungen. Meist scheinen sie der Kontrolle der Kinder zu dienen, um sie in eine Richtung zu weisen oder um eine bestimmte Ordnung, wie zum Beispiel einen Kreis, herzustellen. Timo spielt mehrmals mit Kindern auf dem Schoß ein Reiterspiel, aber immer nur kurz, bis er sich jemand anderem zuwenden muss. Die Berührungen der Kinder lässt Timo zu. Er interagiert dabei nicht, sondern lässt die Kinder auf sich herumturnen. Um Kinder zu trösten, bietet Timo ihnen an, auf die schmerzende Stelle zu pusten oder er versucht die Kinder mit Vorschlägen, was sie alles machen könnten, vom Weinen abzulenken. Er macht sich in einer Situation mit einem anderen Mitarbeitenden lustig über das weinerliche Verhalten eines Kindes.

\subsection{Doing different masculinities}

Wie die Fallbeispiele eindrücklich zeigen, werden Männlichkeiten auf ganz unterschiedliche Art und Weise hergestellt. Um dies noch genauer zu zeigen, werden in diesem Abschnitt zuerst verschiedene Männlichkeiten in vergleichbaren Momenten analysiert. Die Handlungsweisen werden den Typen von Männlichkeiten nach Buschmeyer (2012) zugeordnet, um dann aufzuzeigen, dass jede untersuchte Person nicht einem bestimmten Typus entspricht, sondern diese fluide je nach 
Situation und abhängig vom Kontext zum Ausdruck bringt - doing masculinity erfolgt episodisch. Betrachtet man dies unter Einbezug eines männlichen Habitus wird anschließend versucht aufzuzeigen, warum jede der beobachteten Fachpersonen hegemoniale Männlichkeit zeigt, wenn auch in unterschiedlichen Momenten.

Momente, in denen Männlichkeiten hergestellt werden, sind Berührungen zwischen den männlichen Betreuenden und den Kindern. Sie finden in unterschiedlichen Situationen statt. Für diesen Vergleich von Interesse sind Momente, in denen die männlichen Betreuenden die Kinder zur Kontrolle berühren, in denen die Kinder die Betreuenden berühren, Situationen der Körperpflege und Momente des Tröstens. Michel, Sebastian und Reto scheinen Berührungen bewusst einzusetzen. In vielen Situationen scheint erkennbar zu sein, aus welchem Grund die Kinder berührt werden. Im Unterschied zu Sebastian und Reto, die Berührungen selten einsetzen, scheint Michel Berührungen oft zur Verhaltenskontrolle der Kinder zu nutzen. Bei Timo ist der Grund für die Berührungen, abgesehen von der Kontrolle, oft schwieriger nachzuvollziehen. Bei seinen Berührungen bleibt häufig unklar, wofür er sie einsetzt. Reto und Sebastian lassen die Berührungen der Kinder nur in Situationen zu, in denen sie ihnen etwas beibringen. Beispiele dafür sind Sebastian beim Geschichtenerzählen oder Reto beim Betrachten von Insekten am Boden. Michel berührt in den untersuchten Sequenzen die Kinder zwar von sich aus oft, wird aber selbst von den Kindern kaum berührt. Auch löst er Berührungen schnell wieder auf. Timo lässt die Kinder auf sich herumturnen, interagiert in diesem Moment aber nicht mit ihnen, sondern lässt die Berührungen einfach zu.

Dieses Beispiel zeigt auf, dass die Körperberührungen zwischen den männlichen Fachpersonen und den Kindern vielfältige Männlichkeiten hervorbringen. Keine der beobachteten männlichen Fachpersonen kann dabei genau einem Konstruktionstypen zugeordnet werden. Als komplizenhaft kann zum Beispiel das Handeln beim Trösten von Timo interpretiert werden. Er eifert hegemonialer Männlichkeit nach, indem er das Bedürfnis nach Trost des Kindes herunterspielt. Das heißt konkret, er bietet an, auf die schmerzende Stelle zu pusten oder macht viele Vorschläge, was man tun könnte, um das Kind abzulenken. Auch das schnelle Auflösen der Berührungen der Kinder von Michel kann als komplizenhaft betrachtet werden. Das Nichtreagieren auf die Bedürfnisse nach Trost und körperlicher Nähe stellen hier einen Ausdruck hegemonialer Männlichkeit im Sinne einer Abgrenzung von Weiblichkeit, wie von Connell (2015) beschrieben, dar.

Im Gegensatz dazu zeigen Michel und Reto bei ihrer Art des Tröstens der Kinder auch nichthegemoniale Männlichkeit. Sie verhalten sich fürsorglich und 
empathisch und spenden den Kindern Nähe. Dies kann in diesem Fall als alternative Männlichkeitsform interpretiert werden. Zum Beispiel nimmt Michel ein Kind schnell zu sich, wenn es sich unwohl fühlt, während Reto länger wartet, bevor er ein Kind tröstet.

Generell fällt beim Vergleich der Fachpersonen auf, dass sich sowohl Michel und Timo als auch Sebastian und Reto jeweils in vielen Punkten ähnlich sind. Die Kitas, in denen sie arbeiten, sind sich in Bezug auf das pädagogische Konzept jeweils sehr ähnlich, was einen Zusammenhang des Konzepts der Kitas mit den Möglichkeiten der Konstruktion von Männlichkeiten männlicher Fachkräfte nahelegt. Während das Spiel- und Raumangebot in den Kitas von Michel und Timo eher als stereotyp weiblich konnotiert beschrieben werden können, finden sich für Sebastian und Reto zahlreiche Szenen, in denen sie Kindern männlich konnotierte Tätigkeiten zeigen und gemeinsam mit ihnen ausführen können. In den anderen Kitas ist eine solche Nischenbildung durch das Setting nicht begünstigt und somit viel schwerer als in den beiden Kitas mit vielfältigen Spielmöglichkeiten herstellbar. Sowohl Timo wie auch Michel kontrollieren die Kinder häufig, sei dies in Spielsituationen ohne Betreuende, indem sie immer wieder in den Raum kommen oder durch Körperberührungen, zum Beispiel am Kopf, wenn sich ein Kind nicht wunschgemäß verhält. Diese Position als moralische Instanz bildet eine Möglichkeit, hegemoniale Männlichkeit zu konstruieren. Dazu gehört auch, Handlungen für die Kinder zu übernehmen. Michel und Timo helfen den Kindern in vielen Situationen, während Reto und Sebastian darauf bedacht sind, die Kinder möglichst viel selbst machen zu lassen. Bei Reto und Sebastian steht erzieherisches Handeln im Vordergrund und bei Michel und Timo betreuendes Handeln. Dies sind auch häufig die Momente, in denen sie Männlichkeiten herstellen: Die Nischenbildung durch das Anleiten männlich konnotierter Tätigkeiten und der Ausdruck der hierarchischen Position durch das Helfen und das Zurechtweisen der Kinder.

Ein anderes Beispiel des Einflusses des Kontexts der Kitas auf das Herstellen von Männlichkeiten ist, dass die männlichen Fachpersonen auch unterschiedliche Fehlerkulturen in Bezug auf das Verhalten der Kinder zeigen. Sehr auffällig ist die gezeigte Fehlerkultur von Timo. Er setzt seine Vorstellungen von richtigem Verhalten durch und bestraft die Kinder, die sich diesen nicht beugen. Lob gibt es nur beim Einhalten der Regeln. Ansonsten wird zurechtgewiesen und bestraft. Dieses Verhalten kann als komplizenhaft interpretiert werden. Auch Michel weist häufig zurecht. Allerdings setzt Michel häufiger seinen Körper ein, um falsches Verhalten zu unterbinden. So setzt er ein Kind, das eine Kreissituation stört, auf seinen Schoß und hält es dort fest, bis es sich beruhigt hat. Im Vergleich zu Timo scheint er Zurechtweisen vor allem einzusetzen, um Ordnung zu bewahren, 
und weniger, um eigene Vorstellungen von Verhalten durchzusetzen. Während bei Timo die Fehlerkultur einen Ausdruck komplizenhafter Männlichkeit (er setzt seine hierarchisch höhere Position und seine Vorstellung von positivem und negativem Verhalten gegenüber den Kindern durch) darstellt, scheint sie bei Michel mehr vom Setting, das viele unübersichtliche Situationen fördert, geprägt. Reto und Sebastian weisen eine positive Fehlerkultur auf. Auch zwischen ihnen sind gewisse Unterschiede zu finden. Beide begründen Zurechtweisungen den Kindern gegenüber immer genau. Sie scheinen aufgrund der Beobachtungen zu versuchen, dass das Kind ihre Handlung nachvollziehen kann. Grundsätzlich führt das Setting ihrer Kitas dazu, dass generell wenig Situationen entstehen, in denen Kinder etwas falsch machen können. Beide bieten alternative Handlungsmöglichkeiten an, wenn das Verhalten eines Kindes andere stört. Sie behandeln das Verhalten der Kinder als Ausdruck seiner Bedürfnisse. Sebastian steht häufig aus einer sitzenden Position auf, wenn er ein Kind zurechtweisen muss. Reto begibt sich dabei auf die Höhe der Kinder und versucht gemeinsam mit ihnen alternative Handlungsmöglichkeiten zu finden. Reto verhält sich je nach Kontext unterschiedlich. So zeigt er sich zum Beispiel beim Nachhausegehen weniger geduldig als beim Spielen. Eine positive Fehlerkultur (Fehler passieren und sind positive Lernmomente) ist nur schwer mit hegemonialer Männlichkeit zu vereinbaren. Eine negative Fehlerkultur (Fehler sind etwas Negatives) kann eine Möglichkeit zur Konstruktion hegemonialer Männlichkeit sein. In Bezug auf die Fehlerkultur zeigt vor allem Timo einen komplizenhaften Männlichkeitstypen, während die anderen eher nach Alternativen suchen.

Die Analyse dieser Beispiele zeigt, dass die von Buschmeyer (2012) beschriebenen Männlichkeitstypen im Material durchaus gefunden werden können. Allerdings lassen sich die Typen wie erwartet weniger einzelnen Fällen, sondern vielmehr einzelnen Konstruktionsmomenten, wie zum Beispiel der Zurechtweisung eines Kindes, zuordnen. Die Fachpersonen setzen unterschiedliche Typen von Männlichkeiten kontextuell fluide und episodisch ein.

Pierre Bourdieu (2012, S. 178) geht in seiner Habitustheorie davon aus, dass der vergeschlechtlichte Habitus tief in der Gesellschaft verankert ist und nur sehr schwer veränderlich ist. Dies zeigt sich auch in der Analyse der körperlichen Praktiken männlicher Fachkräfte in Kindertagesstätten. Jeder der untersuchten Fälle zeigt den Einfluss der als soziale Norm verstandenen hegemonialen Männlichkeit (männlicher Habitus) auf die Konstruktion seiner Männlichkeiten. So lassen sich zum Beispiel im Körperausdruck aller männlichen Fachpersonen Elemente komplizenhafter Männlichkeit finden. Komplizenhaft insofern, als dass alle aus einer beobachtenden Position als hegemonial männlich wahrgenommen werden 
können. In der direkten Arbeit mit den Kindern zeigt nur Timo in seinem Ausdruck offensichtlich hegemoniale Elemente. So erscheint er teilweise gelangweilt, indem er zum Beispiel mit den Händen in den Hosentaschen zwischen den Kindern steht, während die Kinder um ihn herumspielen. Er positioniert sich häufig in einer erhöhten Position, indem er zum Beispiel aufrecht steht, während die Kinder am Boden spielen. Die Kinder müssen zu ihm kommen, wenn sie etwas wollen, er bewegt sich nicht von sich aus. Seine Körpersprache macht zum Teil den Anschein, er wolle gar nicht bei den Kindern sein und er sei gelangweilt. Die anderen drei Fälle zeigen hegemoniale Ausdrücke eher in Situationen, in denen sie nicht mit den Kindern arbeiten.

Der männliche Habitus drückt sich unter anderem auch in den Körperkontakten zwischen männlichen Fachpersonen und Kindern aus. Sie sind geprägt von einem hohen Grad an Professionalität und Zweckgebundenheit. Beides stellt für männliche Fachpersonen eine Möglichkeit dar, Berührungen in Anbetracht hegemonialer Männlichkeit zu legitimieren. So machen alle männlichen Fachpersonen in pflegerischen Momenten (zum Beispiel dem Einreiben von Sonnencrème) einen professionellen Eindruck. Sie vermitteln der beobachtenden Person, dass alle Handgriffe eingeübt sind und üben sie auch mit offensichtlicher Selbstverständlichkeit aus. Dieser Eindruck wird dadurch verstärkt, dass auch die Kinder in solchen Situationen genau zu wissen scheinen, wie sich die Betreuenden verhalten werden und was von ihnen jeweils erwartet wird. Bei solchen, aufgrund der pflegerischen Funktion eher weiblich konnotierten Handlungen, scheint Professionalität eine gute Möglichkeit zu sein, sie unter Anbetracht ihrer Männlichkeiten legitim auszuführen. Zum männlichen Habitus gehört unter anderem die Erwartung, bei der Arbeit professionell und leistungsfähig zu sein. Somit bietet möglichst professionelles Verhalten eine Möglichkeit komplizenhaften Verhaltens.

Wie aus den bisherigen Ausführungen ersichtlich wird, beeinflussen die Kontexte, in denen sich die männlichen Fachkräfte bewegen, die möglichen Konstruktionsmomente. So ist eine Herstellung von Männlichkeiten durch die Ausführung männlich konnotierter Tätigkeiten für gewisse Einzelfälle aufgrund des Kontexts (die Settings der Kitas lassen keine oder wenig männlich konnotierte Tätigkeiten zu) schwieriger als für die Fälle, die in ihrem Arbeitsalltag bereits eine Vielzahl an Möglichkeiten für männlich konnotierte Tätigkeiten vorfinden. So lässt zum Beispiel die Kita, in der Michel arbeitet, aufgrund der Räumlichkeiten und des Spielangebots nicht zu, dass er etwas Handwerkliches mit den Kindern erarbeitet, während die Kita, in der Sebastian arbeitet, über einen großzügigen Bastel- und Werkraum verfügt.

Die beobachtete hegemoniale Männlichkeit kann somit episodisch unterschiedlich hergestellt werden. In den durch die Tätigkeit bereits männlich konnotierten 
Momenten reicht es aus, die Tätigkeit auszuführen, während in weiblich konnotierten Momenten eine entsprechend hegemonialer Männlichkeit vorgenommene Interpretation der Tätigkeit gezeigt wird. Zum Beispiel kümmert sich Timo vor allem darum, während des Spielens der Kinder verschiedene Gegenstände, die kaputt gehen, zu reparieren. Aber nicht nur das Raum- und Spielangebot hat einen Einfluss auf die Möglichkeiten zur Herstellung von Männlichkeiten. In den beobachteten Fällen könnte das gezeigte Verhalten auch mit den unterschiedlichen pädagogischen Konzepten der Kitas zu tun haben. So führen die oft entstehenden, unübersichtlichen Momente, in denen viele Kinder gleichzeitig unterschiedliche Tätigkeiten ausführen dazu, dass die Kinder häufiger an- und zurechtgewiesen werden müssen. Entsprechend müssen auch die Betreuenden viel Aufwand betreiben, um Regeln und eine gewisse Ordnung durchzusetzen. Dies führt zu mehr Situationen, in denen die Hierarchie zwischen Kindern und Betreuenden zum Ausdruck kommt. Und dies wiederum bietet eine Möglichkeit zur Herstellung hegemonialer Männlichkeit. Eine weitere Möglichkeit bietet sich durch professionelles Handeln. Wie bereits Buschmeyer (2012, S. 237) feststellte, legitimieren männliche Fachpersonen in Kindertagesstätten ihre Männlichkeiten über einen möglichst hohen Grad an Professionalität. Dies zeigt sich auch in den untersuchten körperlichen Handlungen, insbesondere in pflegerischen Handlungen mit Körperkontakt. Alle untersuchten Situationen, in denen Kinder durch männliche Betreuende gepflegt werden, zeichnen sich durch keinerlei Unsicherheiten vonseiten der Fachpersonen aus und ihr Vorgehen erscheint professionell erlernt und routiniert.

\section{7 (Un)doing masculinity in der rekonstruktiven Videoanalyse}

Die Ergebnisse dieser Untersuchung zeigen die Allgegenwärtigkeit und Wirkmächtigkeit von inkorporierten, hegemonialen Männlichkeitsstrukturen. Jeder der untersuchten männlichen Fachkräfte zeigt in unterschiedlichen Momenten, dass auch in seinem Habitus hegemoniale Muster vorhanden sind. Die Analyse zeigt, dass die von Connell und Buschmeyer entwickelten Typen von Männlichkeiten im Material durchaus gefunden werden können. Allerdings lassen sich die Typen weniger einzelnen Fällen, sondern vielmehr episodisch einzelnen Konstruktionsmomenten zuordnen. Der jeweilige Kontext der Kita, in dem sich die männlichen Fachkräfte bewegen, hat einen starken Einfluss auf die möglichen Konstruktionsmomente. Bestehen in der Kita wenig Möglichkeiten zur Bildung von Nischen, drückt sich hegemoniale Männlichkeit vor allem im Umgang mit den Kindern 
aus - spezifisch in Momenten, in denen die Kinder zurechtgewiesen werden. Die hierarchischen Strukturen zwischen Betreuenden und Kindern bieten weitere Möglichkeiten der Konstruktion von Männlichkeiten.

Die Videoanalyse hat verglichen mit klassischeren Auswertungsmethoden, wie zum Beispiel Interviews, einige Vorteile. So lässt sich mit Videodaten die soziale Praxis sehr präzise rekonstruieren. Ethnografische Methoden eignen sich in besonderer Weise. Die Videoanalyse ermöglicht es Forschenden, Erhebung und Interpretation der Daten klar zu trennen und die Ergebnisse sind einfacher zu reproduzieren und zu reflektieren. Besonders für die Untersuchung von non-verbalen Handlungen sind Videobeobachtungen anderen Erhebungsmethoden vorzuziehen. Allerdings muss auch hier kritisch betrachtet werden, dass das Video trotzdem kein genaues Abbild der Realität darstellt, da es durch die Anwesenheit der Forschenden wie auch der Kamera beeinflusst ist. Zudem lassen sich Faktoren wie Gerüche nicht rekonstruieren. Die methodologische Reflexion steht erst am Anfang und es ist somit davon auszugehen, dass viele problematische Aspekte dieser Analysen noch nicht genauer erfasst sind. Umso wichtiger erscheint das systematisch erstellte Forschungstagebuch. Es trägt dazu bei, die subjektive Wahrnehmung des Forschenden zu reflektieren. Allerdings kann es den Qualitätsgewinn eines größeren Forschungsteams für eine objektivere Analyse nicht ersetzen. Zwar wurde das Vorgehen mit verschiedenen anderen Fachpersonen reflektiert, es würden aber weitere forschende Personen benötigt, die sich auf der gleichen Ebene mit dem Material beschäftigen.

Bourdieu (2012) beschreibt in seinem Werk zur männlichen Herrschaft diese unüberwindbar scheinenden Strukturen der geschlechtlichen Dualität: „In der Tat hat es etwas Erstaunliches, dass die geschlechtlichen Strukturen gegenüber den ökonomischen Strukturen, die Reproduktionsweisen gegenüber den Produktionsweisen diese außerordentliche Autonomie besitzen" (Bourdieu 2012, S. 142). Ein undoing gender wird aus dieser Perspektive ungleich schwieriger, muss sich doch die etablierte Struktur immer wieder neu in Szene setzen. Die hier eingenommene Analyseperspektive körperlicher Praktiken fokussiert somit auch spezifisch auf ein undoing gender im Sinn von undoing hegemonic masculinity, also auf das undoing einer hegemonialen Form von Männlichkeit und wie sie in der Kita inszeniert werden.

Wie in den Ergebnissen ausführlich beschrieben, ist ein Aspekt dieser Strukturmacht auch der augenfällige Einfluss des Kontexts auf die Möglichkeiten der männlichen Fachpersonen, Männlichkeiten zu konstruieren. In den Kitas mit eher weiblich konnotierten Räumen und Spielangeboten konstruieren die beobachteten männlichen Betreuenden hegemoniale Männlichkeit vor allem in der Interaktion mit den Kindern. In den Kontexten, die eine Bandbreite an männlich und 
weiblich konnotierten Tätigkeiten zulassen, stellen die untersuchten Fachpersonen hegemoniale Männlichkeit vor allem durch die Ausübung männlich konnotierter Tätigkeiten, wie zum Beispiel Holzhacken, her. Der Kontext beeinflusst also die Möglichkeiten der Konstruktion von Männlichkeiten. So lässt zum Beispiel ein veränderbares Setting im Wald mehr unterschiedlich konnotierte Aktivitäten zu als Räumlichkeiten, die hauptsächlich von den Fachpersonen gestaltet und unterhalten werden. Bestehende Konzepte und Settings in der Praxis sollten also bezüglich ihrer episodischen Konstruktionsmomente hegemonialer Männlichkeit überprüft und falls notwendig angepasst werden.

Neben der Sichtweise, dass der Kontext die Handlungsmöglichkeiten der Fachpersonen beeinflusst, stellt Buschmeyer (2012, S. 274) aber auch fest, dass Fachpersonen je nach gezeigten Männlichkeitskonstruktionen eher in Einrichtungen arbeiten, die diesen förderlich sind (Buschmeyer 2012). Betrachtet man die hier untersuchten Personen mit dieser Sichtweise, entsteht das Bild, dass die Fachpersonen mit mehr als komplizenhaft eingestuften Praktiken eher in Einrichtungen mit weiblich konnotierten Einrichtungsgegenständen arbeiten. Fachpersonen mit mehr alternativ eingestuften Praktiken sind eher in Einrichtungen beschäftigt, die eine breite Palette an unterschiedlich konnotierten Tätigkeiten ermöglichen.

Auch in der hier vorliegenden Analyse wird die Nischenbildung männlicher Fachpersonen als Möglichkeit bestätigt, hegemoniale Männlichkeit zu konstruieren. In Anbetracht der Konstruktion hegemonialer Männlichkeit bei allen untersuchten Fällen lässt sich vermuten, dass die Ausführung von männlich konnotierten Tätigkeiten den untersuchten männlichen Fachpersonen ermöglichen könnte, in der Interaktion mit den Kindern alternative Männlichkeit zu leben. Männliche Fachpersonen ohne diese Möglichkeit andere Momente für die Konstruktion hegemonialer Männlichkeit suchen. So zeigen die beiden Fachpersonen, die männlich konnotierte Tätigkeiten (wie zum Beispiel Holz hacken, Feuer machen, mit scharfen Messern schneiden etc.) mit den Kindern ausüben, ansonsten keine weiteren als hegemonial männlich eingestufte Momente. Die beiden Fälle, in denen solche Tätigkeiten nicht möglich sind, zeigen vermehrt hegemoniale Männlichkeitskonstruktionen, zum Beispiel im Umgang mit den Kindern, indem sie vermehrt ihre hierarchische Position den Kindern gegenüber hervorheben (zum Beispiel durch Zurechtweisen etc.).

Buschmeyer (2012) empfiehlt in ihren Schlussfolgerungen, man solle in zukünftigen Forschungen nicht mehr generell von doing masculinity, sondern von doing hegemonic masculinity, doing complicit masculinity und doing alternative masculinity sprechen. Dies würde zum Verständnis beitragen, dass männliche Fachpersonen nicht immer nach dem gleichen Typus handeln, sondern situationsbedingt andere Konstruktionstypen zeigen und diese fluide und episodenhaft 
einsetzen können (Buschmeyer 2012, S. 278). Die Ergebnisse der vorliegenden Untersuchung unterstützen die Überlegungen Buschmeyers. Unter Einbezug des männlichen Habitus zeigt sich klar, dass aufgrund der tiefen gesellschaftlichen Verankerung nicht von (un)doing gender sondern von (un)doing hegemonic masculinity gesprochen werden muss.

$\mathrm{Zu}$ einem ähnlichen Befund kommen auch Tennhoff (2018) sowie die in Kap. 6 dargestellte Analyse der Subjektivierungspraktiken männlicher Fachpersonen. Keine der Fachpersonen kommt ganz ohne einen Verweis auf hegemoniale Männlichkeit aus, die Praktiken unterliegen aber auch hier einer situativen Wechselhaftigkeit und je nach argumentativer Situation werden unterschiedliche Positionen eingenommen.

Diese Erkenntnisse eröffnen neue Handlungsmöglichkeiten und Perspektiven. So sollte man im Anschluss an diese Untersuchungen in Forschung und Praxis nicht mehr der Frage nachgehen, welche Männlichkeitstypen sich besonders für die Arbeit in Kindertagesstätten eignen, sondern vielmehr, welche Situationen episodisch welche Arten der Konstruktion von Männlichkeiten zulassen. Man könnte für die Praxis Momente aufzeigen, die nach besonderer Reflexion von Geschlechterstereotypen verlangen. Dies wäre nicht nur für die Emanzipation unterschiedlicher Männlichkeiten (Buschmeyer 2012, S. 277) und die Entstereotypisierung des Erziehendenberufs als weiblich konnotiertem Arbeitsfeld, sondern darüber hinaus auch für die Anerkennung des Berufs im Allgemeinen förderlich. Um alternative Männlichkeitsformen in Kitas konkret zu ermöglichen, könnten zum Beispiel mehr Nischen zur Konstruktion hegemonialer Männlichkeit geschaffen werden. Die Untersuchung zeigt, dass solche Nischen zum Beispiel ein Setting im Wald oder Bastel- und Werkmöglichkeiten ermöglichen können. Dadurch eröffnen sich viele unterschiedlich konnotierte Tätigkeiten. Im pädagogischen Handeln sollte insbesondere auch die Fehlerkultur in den Blick gerückt werden; wie wird in einer Kita mit Fehlern und als störend ausgelegtem Verhalten der Kinder umgegangen. Und schlussendlich ist es wichtig, auch die hierarchischen Strukturen zwischen Betreuenden und Kindern und unter Betreuenden genau zu analysieren. Sind zum Beispiel männliche Fachpersonen auf allen Hierarchiestufen vorhanden oder sind sie nur in Führungspositionen? Stellt sich eine Kita regelmäßig solche Fragen, ermöglicht sie es ihren männlichen Mitarbeitenden eher, alternative Formen von Männlichkeiten zu leben. 


\section{Literatur}

Blaschke, Gerald. (2012). Schule schnuppern: eine videobasierte Studie zum Übergang in die Grundschule. Opladen, Berlin \& Toronto: Barbara Budrich.

Bourdieu, Pierre. (2012). Die männliche Herrschaft. Frankfurt am Main: Suhrkamp Verlag.

Brandes, Holger. (2004). Hegemoniale Männlichkeit und männlicher Habitus. Thesen zu Connell und Bourdieu. Arbeitskreis (2001), aaO.

Buschmeyer, Anna. (2012). Zwischen Vorbild und Verdacht: Wie Männer im Erzieherberuf Männlichkeit konstruieren. Wiesbaden: VS Verlag für Sozialwissenschaften.

Connell, Reawyn. (2015). Der gemachte Mann: Konstruktion und Krise von Männlichkeiten (4. Auflage). Wiesbaden: VS Verlag für Sozialwissenschaften.

Gildemeister, Regine. (2017). Doing Gender: eine mikrotheoretische Annäherung an die Kategorie Geschlecht. In Beate Kortendiek, Birgit Riegraf, \& Katja Sabisch (Hrsg.), Handbuch Interdisziplinäre Geschlechterforschung (Vol. 65, pp. 1-9). Wiesbaden: VS Verlag für Sozialwissenschaften.

Kelle, Udo, \& Kluge, Susann. (2010). Vom Einzelfall zum Typus: Fallvergleich und Fallkontrastierung in der qualitativen Sozialforschung (2. Auflage). Wiesbaden: VS Verlag für Sozialwissenschaften.

Meuser, Michael. (2006). Hegemoniale Männlichkeit-Überlegungen zur Leitkategorie der men's studies. In Brigitte Aulenbacher, Mechthild Bereswill, Martina Löw, Michael Meuser, Gabriele Mordt, Reinhild Schäfer, \& Sylka Scholz (Hrsg.), FrauenMännerGeschlechterforschung: state of the art (S. 160-171). Münster: Westfälisches Dampfboot.

Rohrmann, Tim. (2006). Männer in Kinderbetreuungseinrichtungen: Bestandsaufnahme und Perspektiven. Beitrag präsentiert an der Fachtagung Mehr Männer in die pädagogische Arbeit! Beitrag verfügbar unter https://wechselspiel-online.de/frameset/frameset_litera tur.html.

Rohrmann, Tim, Cremers, Michael, \& Krabel, Jens. (2010). Männer in Kitas - welche Bedeutung hat das Geschlecht pädagogischer Fachkräfte. Archiv für Wissenschaft und Praxis der sozialen Arbeit, 2, 1-12.

Tennhoff, Wiebke. (2018). Symbolische Väter, junge Wilde und professionelle Pädagogen. Diskursive Konstruktionen männlicher Geschlechtsidentität in Kindertageseinrichtungen (Dissertation). Universität St. Gallen. Verfügbar unter https://www.alexandria.unisg.ch/ 257653/

Wagner-Willi, Monika. (2013). Videoanalysen des Schulalltags: Die dokumentarische Interpretation schulischer Übergangsrituale. In Ralf Bohnsack, Iris Nentwig-Gesemann, \& Arnd-Michael Nohl (Hrsg.), Die dokumentarische Methode und ihre Forschungspraxis: Grundlagen qualitativer Sozialforschung (3. Auflage, S. 133-177). Wiesbaden: VS Verlag für Sozialwissenschaften.

Wilz, Sylvia. (2002). Organisation und Geschlecht: Strukturelle Bindungen und kontingente Koppelungen. Wiesbaden: VS Verlag für Sozialwissenschaften. 
Open Access Dieses Kapitel wird unter der Creative Commons Namensnennung 4.0 International Lizenz (http://creativecommons.org/licenses/by/4.0/deed.de) veröffentlicht, welche die Nutzung, Vervielfältigung, Bearbeitung, Verbreitung und Wiedergabe in jeglichem Medium und Format erlaubt, sofern Sie den/die ursprünglichen Autor(en) und die Quelle ordnungsgemäß nennen, einen Link zur Creative Commons Lizenz beifügen und angeben, ob Änderungen vorgenommen wurden.

Die in diesem Kapitel enthaltenen Bilder und sonstiges Drittmaterial unterliegen ebenfalls der genannten Creative Commons Lizenz, sofern sich aus der Abbildungslegende nichts anderes ergibt. Sofern das betreffende Material nicht unter der genannten Creative Commons Lizenz steht und die betreffende Handlung nicht nach gesetzlichen Vorschriften erlaubt ist, ist für die oben aufgeführten Weiterverwendungen des Materials die Einwilligung des jeweiligen Rechteinhabers einzuholen.

(c) (1) 